\title{
A COMPUTATIONALLY EFFICIENT AND ACCURATE NUMERICAL REPRESENTATION OF THERMODYNAMIC PROPERTIES OF STEAM AND WATER FOR COMPUTATIONS OF NON-EQUILIBRIUM CONDENSING STEAM FLOW IN STEAM TURBINES
}

\begin{abstract}
Jan HRUBÝ•
Abstract: Mathematical modeling of the non-equilibrium condensing transonic steam flow in the complex 3D geometry of a steam turbine is a demanding problem both concerning the physical concepts and the required computational power. Available accurate formulations of steam properties IAPWS-95 and IAPWS-IF97 require much computation time. For this reason, the modelers often accept the unrealistic ideal-gas behavior. Here we present a computation scheme based on a piecewise, thermodynamically consistent representation of the IAPWS-95 formulation. Density and internal energy are chosen as independent variables to avoid variable transformations and iterations. On the contrary to the previous Tabular Taylor Series Expansion Method, the pressure and temperature are continuous functions of the independent variables, which is a desirable property for the solution of the differential equations of the mass, energy, and momentum conservation for both phases.
\end{abstract}

\section{INTRODUCTION}

Realistic computation of the flow of the condensing steam in a steam turbine is a prerequisite of development of more reliable and efficient turbines. Realistic computational fluid dynamics (CFD) model also includes properties of real steam. In a finite-volume computation, thermodynamic properties need to be evaluated several times in each volume element and each time step. Consequently, besides accuracy, the mathematical model must also be computationally efficient. The lack of such models is one of the reasons that often the real gas behavior is neglected and only the ideal gas equation is considered, despite a large error due to neglecting the real gas properties.

In this work we focus on thermodynamic properties of single-phase steam, either in stable states - superheated or saturated steam - or in metastable states supersaturated (subcooled) steam.

The finite-volume model solves balances of mass, momentum and energy in a volume element. From these balances, one obtains the mass density $\rho$ and the specific internal energy $u$. For these variables it is necessary to determine pressure $p$ in order to evaluate the momentum fluxes through the finite-volume boundaries, and temperature $T$ to determine heat fluxes at walls. Temperature and pressure (or density) are further used to determine transport properties (viscosity, thermal conductivity) and the nucleation

\footnotetext{
'Institute of Thermomechanics AS CR, v.i.i. Dolejškova 5, Prague 8, Czech Republic

hruby@it.cas.cz
}

This is an Open Access article distributed under the terms of the Creative Commons Attribution License 2.0, which permits unrestricted use, distribution, and reproduction in any medium, provided the original work is properly cited. 
rate. The numerical solver might require computation of other thermodynamic properties, e.g. speed of sound.

The International Association for the Properties of Water and Steam (IAPWS) developed a widely accepted scientific formulation of thermodynamic properties of steam known as IAPWS-95 [1,2]. This formulation is based on correlating all available data for thermodynamic properties of water and steam and it is in the fundamental form of Helmholtz energy with independent variables density and temperature in dimensionless form. Because this formulation is rather complex, the so-called industrial formulation IF$97[3,4]$ was developed on the basis of the IAPWS-95 scientific formulation. IF-97 is in the range of interest - so-called region 2 - formulated as the Gibbs energy in variables pressure and temperature. These variables are advantageous for many practical purposes. However, for CFD computations neither of these formulations is suitable, because the different variables would require time-consuming iterations. Moreover, both formulations are valid only in the stable region. In the supersaturated steam region, they exhibit unrealistic oscillations. For supersaturated steam, a separate equation is provided by the IF-97 and the IAPWS-95 should be replaced by the so-called gas equation [1]. The change of equation when crossing the equilibrium saturation line is uncomfortable for CFD programmers and also the small inconsistencies of the equations for the stable and metastable region may cause numerical problems.

There are essentially two ways of representing thermodynamic properties. The first one is by a global analytical equation. The second is the so-called table look-up. The second way may provide very quick evaluations of thermodynamic properties. The fact that it requires a larger memory capacity (order of $10 \mathrm{MB}$ ) is not a difficulty for present machines. Real trouble of the available methods is discontinuity at the borders between the tabulated cells. The CFD solvers assume some level of smoothness (continuity of derivatives up to certain order). The discontinuities cause a quasi-random noise which can deteriorate the performance of the solver or even lead to lack of convergence or unphysical oscillations. An advanced table look up method was developed by Miyagawa and Hill $[5,6]$. It is based on the second-order Taylor expansion of a thermodynamic potential with respect to two variables (e.g. density and temperature [6]). Six coefficients of the expansion are tabulated for each cell, enabling computation of any thermodynamic property.

A global analytical formulation, on the other hand, provides differentiability of arbitrary order at price of higher computation costs. At present, no global formulation is available for density and internal energy as independent variables. Advantages of both approaches may, to a certain extent, be combined in a spline-interpolation method, providing continuity and smoothes at the borders of tabulated cells (patches).

An important aspect is the thermodynamic consistency of the formulations. Thermodynamic consistency is assured by fundamental formulations, in which all thermodynamic properties are obtained from a single function (e.g. Helmoltz function depending on density and temperature) by using general thermodynamic identities.

In this work we develop a hybrid approach to formulation of thermodynamic properties of steam, combining the spline method in the $u$-direction with a global fit in the $\rho$-direction. Thermodynamically consistent model is obtained by considering entropy as function of these variables as a fundamental function, from which all thermodynamic properties are obtained using thermodynamic relations. 


\section{Structure Of the NeW Formulation}

The new formulation is based on function

$$
s(u, \rho),
$$

i.e. specific entropy as function of specific internal energy and specific density. The first law of thermodynamics for a reversible change can be written as

$$
d s=\frac{1}{T} d u-\frac{p}{T \rho^{2}} d \rho .
$$

Since Eq. (2) represents a total differential of function (1), the following relations hold:

$$
\begin{gathered}
s_{u} \equiv\left(\frac{\partial s}{\partial u}\right)_{\rho}=\frac{1}{T}, \\
s_{\rho} \equiv\left(\frac{\partial s}{\partial \rho}\right)_{u}=-\frac{p}{T \rho^{2}} .
\end{gathered}
$$

Once function (1) is established, temperature and pressure can be obtained for given density and internal energy as

$$
\begin{gathered}
T=\frac{1}{s_{u}}, \\
p=-T \rho^{2} s_{\rho} .
\end{gathered}
$$

Other thermodynamic properties are obtained using general thermodynamic relations. For example, enthalpy is found as

$$
h=u+p / \rho
$$

using pressure obtained from Eq. (6). Some thermodynamic properties require secondorder partial derivatives

$$
s_{u u} \equiv\left(\frac{\partial^{2} s}{\partial u^{2}}\right)_{\rho}, \quad s_{u \rho} \equiv \frac{\partial^{2} s}{\partial u \partial \rho}, \quad s_{\rho \rho} \equiv\left(\frac{\partial^{2} s}{\partial \rho^{2}}\right) .
$$

For example, the isochoric heat capacity is obtained as

$$
c_{v}=-\frac{1}{T s_{u u}}
$$

and the square of the speed of sound is found as

$$
a^{2}=\left(\frac{\partial p}{\partial \rho}\right)_{s}=-2 p T s_{u \rho}-\frac{p^{2} T}{\rho^{2}} s_{u u}-T \rho^{2} s_{\rho \rho}+\frac{2 p}{\rho} .
$$

Entropy (1) is separated into three terms: ideal gas entropy $s_{\text {ref }}^{\text {id }}$ at the reference density $\rho_{\text {ref, }}$ change of ideal gas entropy due to actual density, and residual entropy $s_{\text {res }}$ expressing the real-gas effect due to molecular interactions: 


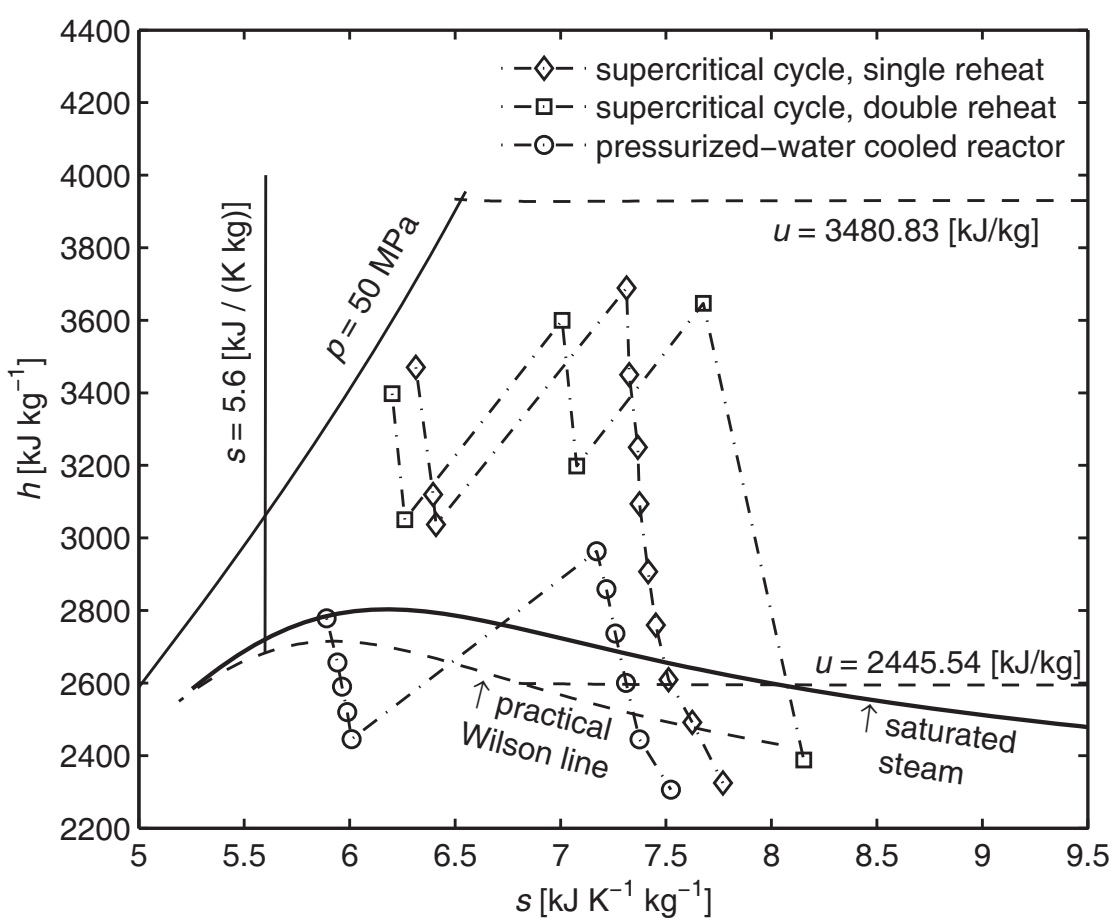

Figure 1: The required range of validity of the formulation in the entropy - enthalpy diagram.

$$
s(u, \rho)=s_{\mathrm{ref}}^{\mathrm{id}}(u)-R \ln \frac{\rho}{\rho_{\text {ref }}}+s^{\mathrm{res}}(u, \rho) .
$$

As indicated in Eq. (11), the ideal-gas entropy at reference density only depends on the internal energy. The detailed forms of the ideal gas term and the residual term will be discussed in separate sections.

\section{RANGE OF VALIDITY}

The formulation is developed to cover completely the operation region of steam turbines, including supercritical cycles and nuclear cycles. It also covers the region of metastable supersaturated steam. The region is shown in Figure 1. The diagram shows expansion lines of typical modern steam cycles. The region of validity is limited by the following relations:

$$
\begin{gathered}
s \geq 5.6 \mathrm{~kJ} \mathrm{~K}^{-1} \mathrm{~kg}^{-1}, \\
p \leq 50 \mathrm{MPa} \\
2445.54 \leq \frac{u}{\mathrm{~kJ} \mathrm{~kg}^{-1}} \leq 3480.83, \\
y \leq 0.05 .
\end{gathered}
$$




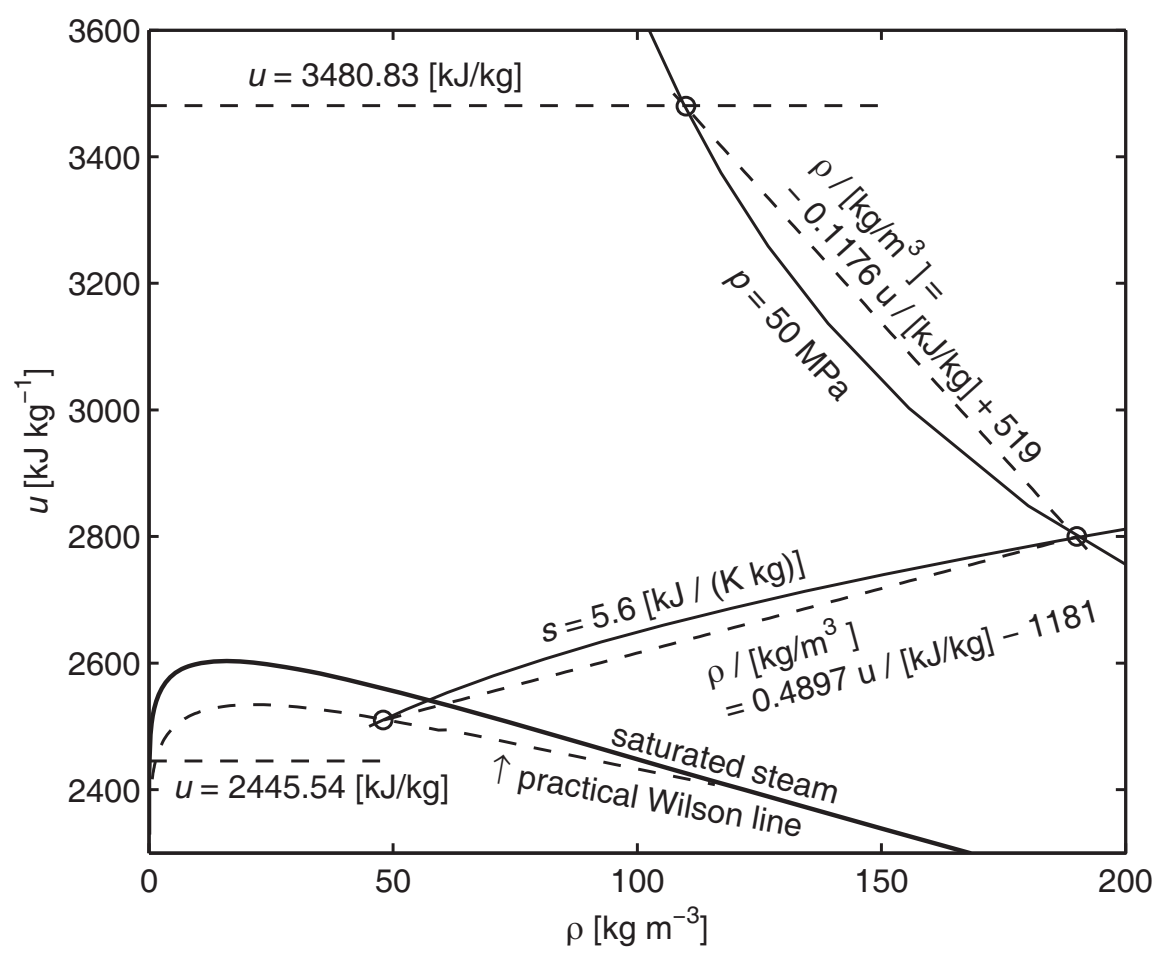

Figure 2: Region of validity in the density-internal energy diagram.

The last equation characterizes the extent to which the supersaturated steam is considered. In reality, the limit of supersaturation is given by the onset of nucleation which, however, depends on particular conditions. When plotted in a Mollier diagram, the locus of nucleation onset is called the Wilson line. In order to avoid the complexity of nucleation in specifying the range of validity, we accept the method given in the industrial formulation IF-97 [4]. The practical Wilson line is determined as line of equilibrium moisture of $5 \%$ in the pressure-enthalpy diagram. For a given pressure $p$ and enthalpy $h$ of the supersaturated steam we compute the corresponding equilibrium moisture $y$ as

$$
y=\frac{h-h^{\prime}(p)}{h^{\prime \prime}(p)-h^{\prime}(p)},
$$

where the saturated liquid enthalpy $h$ ' and the saturated vapor enthalpy $h$ ' are obtained for the actual pressure of the supersaturated steam.

The range of internal energies (14) corresponds in the zero-density limit to temperatures $50^{\circ} \mathrm{C}$ and $700^{\circ} \mathrm{C}$.

Figure 2 shows the region of validity in the variables of the formulation, density and internal energy. It was practical to replace the entropy limit given by Eq. (12) by a straight line

$$
\frac{\rho}{\mathrm{kg} \mathrm{m}^{-3}} \leq 0.4897 \frac{u}{\mathrm{~kJ} \mathrm{~kg}^{-1}}-1181
$$




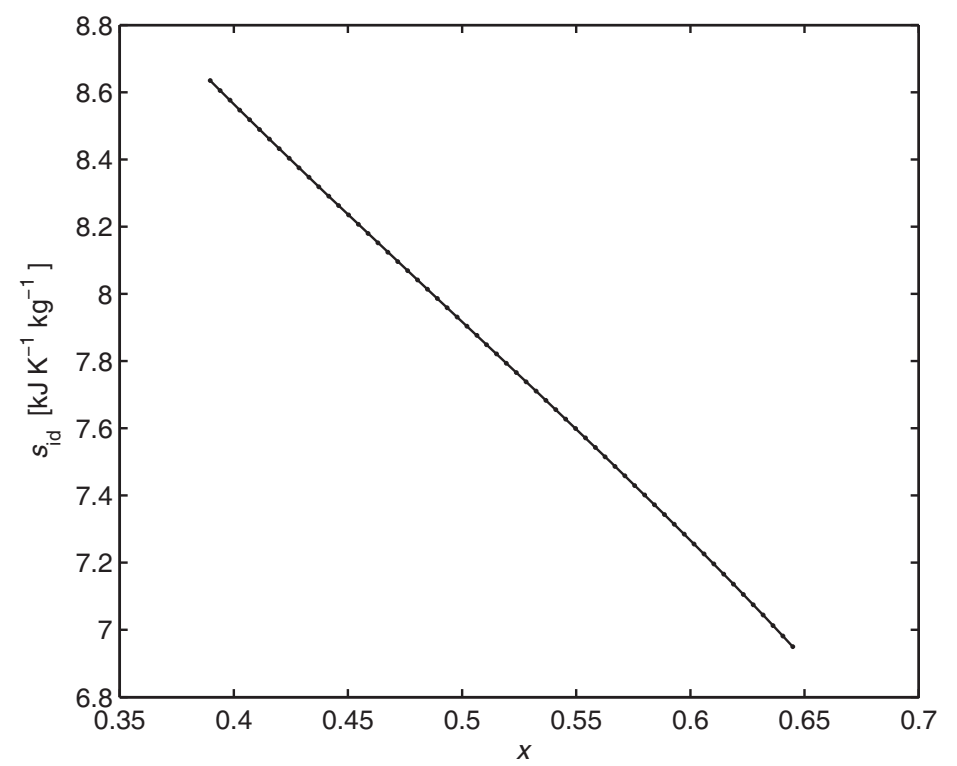

Figure 3: Ideal-gas entropy as function of variable $x$, given by Eq. (19)

Similarly the limitation to pressures up to $50 \mathrm{MPa}$, Eq. (13), was replaced by a straight line

$$
\frac{\rho}{\mathrm{kg} \mathrm{m}^{-3}} \leq 0.1176 \frac{u}{\mathrm{~kJ} \mathrm{~kg}^{-1}}+519
$$

\section{Ideal Gas Properties}

The ideal gas entropy at reference density $\rho_{\text {ref }}=1 \mathrm{~kg} \mathrm{~m}^{-3}$ was generated from the IAPWS-95 formulation. We represented the generated data in form of a quadratic spline with continuous first derivatives. Using the internal energy as interpolation variable brought about a large scatter of the derivatives. Therefore, we introduced an energy parameter $x$,

$$
x \equiv \frac{10^{6}}{u /\left[\mathrm{J} \mathrm{kg}^{-1}\right]-9 \times 10^{5}} .
$$

As shown in Figure 3, the dependence of the ideal gas entropy on this parameter is an almost linear function, which is an advantageous feature for interpolation. The figure also shows the knots of the interpolation. The interval on the $x$-axis, corresponding to the range of internal energies, was divided into 60 equal subintervals, on which the entropy was represented by a smooth piecewise quadratic function. Temperature, given by the first derivative, Eq. (5), is continuous, but not smooth. The deviations of the interpolated temperatures from the accurate ones are shown in Figure 4. Numerically these deviations remain small, about $\pm 0.01 \mathrm{~K}$. Properties depending on second derivatives are not even continuous. However, their accuracy is sufficient. In particular, the speed of sound obtained with Eq. (10) is accurate within $0.1 \%$. 


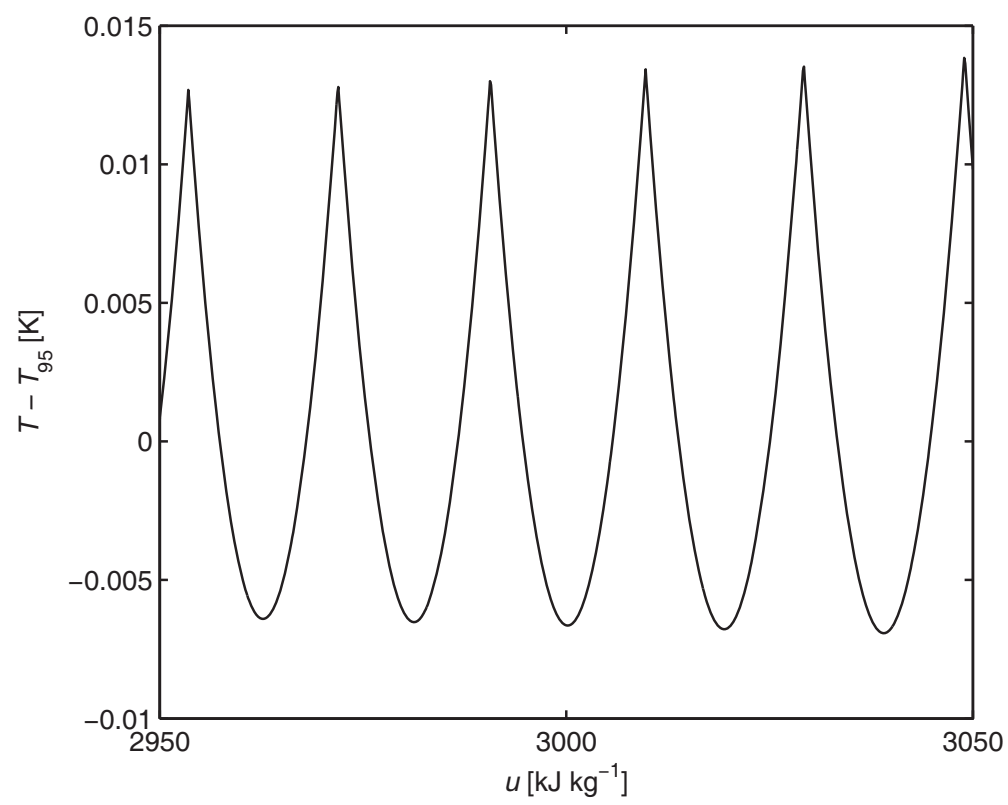

Figure 4: Deviation of the spline-interpolated temperature from the accurate value generated from the IAPWS-95 formulation

\section{RESIDUAL ENTROPY FunCtion}

In order to develop an appropriate expression for the residual entropy, we first analyzed the dependence compressibility factor

$$
Z \equiv \frac{p}{\rho R T}
$$

on density for fixed values of internal energy, as shown in Figure 5. Data produced from the gas equation [1] were used for density up to $55 \mathrm{~kg} \mathrm{~m}^{-3}$ and the IAPWS-95 formulation was used for higher densities. The compressibility factor was expanded into a truncated virial series

$$
Z=1+\sum_{i=1}^{7} a_{i} \delta^{i}
$$

where $\delta \equiv \rho / \rho_{c}$ and $\rho_{c}=322 \mathrm{~kg} \mathrm{~m}^{-3}$ is the density of water at the critical point. The virial coefficients $a_{i}$ have alternating sings, starting from a negative $a_{1}$. The coefficients very strongly increase with decreasing internal energy (corresponding to decreasing temperature at zero density). In order to provide an accurate spline-interpolation scheme, we scaled these dependencies as shown in Figure 6. The integer powers $n_{i}$ were chosen such that the transformed coefficients $A_{i}$, defined as

$$
A_{i} \equiv\left[(-1)^{i} a_{i}\right]^{-1 / n_{i}},
$$

are almost linear functions of the energy parameter $x$ defined by Eq. (19). The linearity was required especially in the region of low internal energies (high $x$ ), where an accurate 


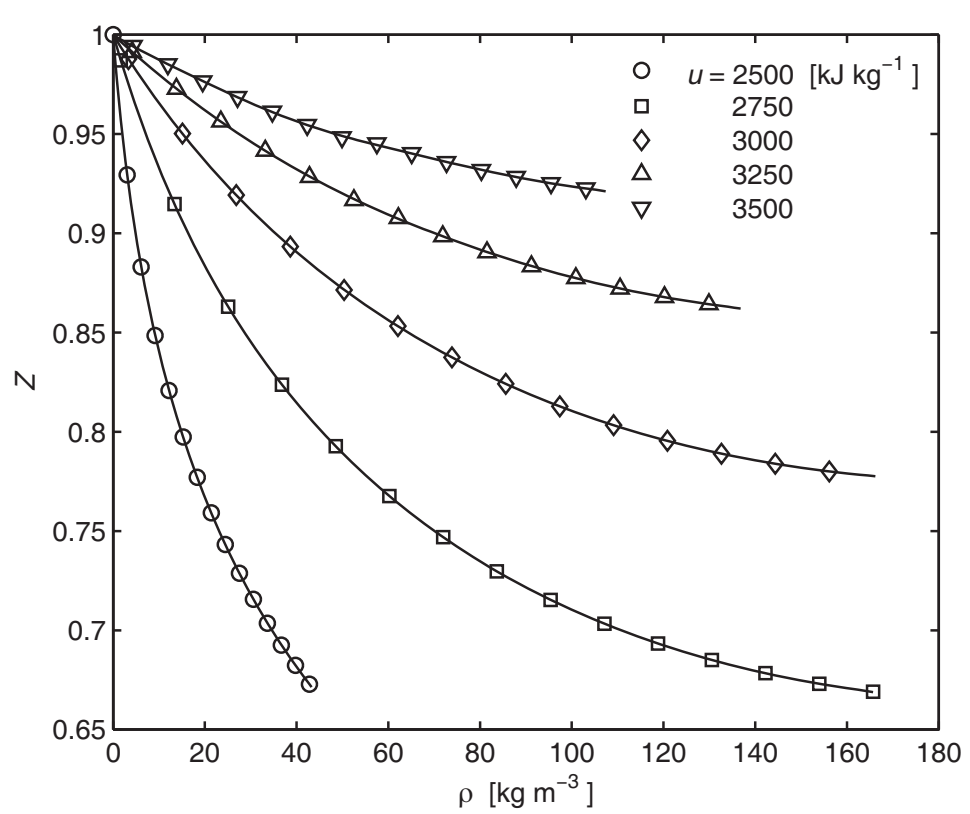

Figure 5: Compressibility factors for various internal energies as functions of density.

interpolation is needed to correctly represent the strong real-gas effects of saturated and supersaturated steam. In computations, the inverse function must be evaluated:

$$
a_{i}=\frac{(-1)^{i}}{A_{i}^{n_{i}}} \text {. }
$$

This evaluation is fast when the integer powers are implemented via multiplication.

Now we develop a formula relating the residual entropy to the compressibility factor given by Eq. (21). From Eq. (11) we find for the dependence of the residual entropy on density

$$
\left(\frac{\partial s^{\mathrm{res}}}{\partial \rho}\right)_{u}=\left(\frac{\partial s}{\partial \rho}\right)_{u}+\frac{R}{\rho} .
$$

Further, using Eq. (4) at place of the entropy derivative and definition (20) of the compressibility factor

$$
\left(\frac{\partial s^{\mathrm{res}}}{\partial \rho}\right)_{u}=-R \frac{Z-1}{\rho} .
$$

In the integration we use the fact that the residual entropy, by definition, vanishes at zero density:

$$
s^{\mathrm{res}}=-R \int_{0}^{\rho} \frac{Z\left(u, \rho^{\prime}\right)-1}{\rho^{\prime}} d \rho^{\prime}=-R \int_{0}^{\delta} \frac{Z\left(u, \delta^{\prime}\right)-1}{\delta^{\prime}} d \delta^{\prime} .
$$

For the truncated virial series given by Eq. (21) we obtain 


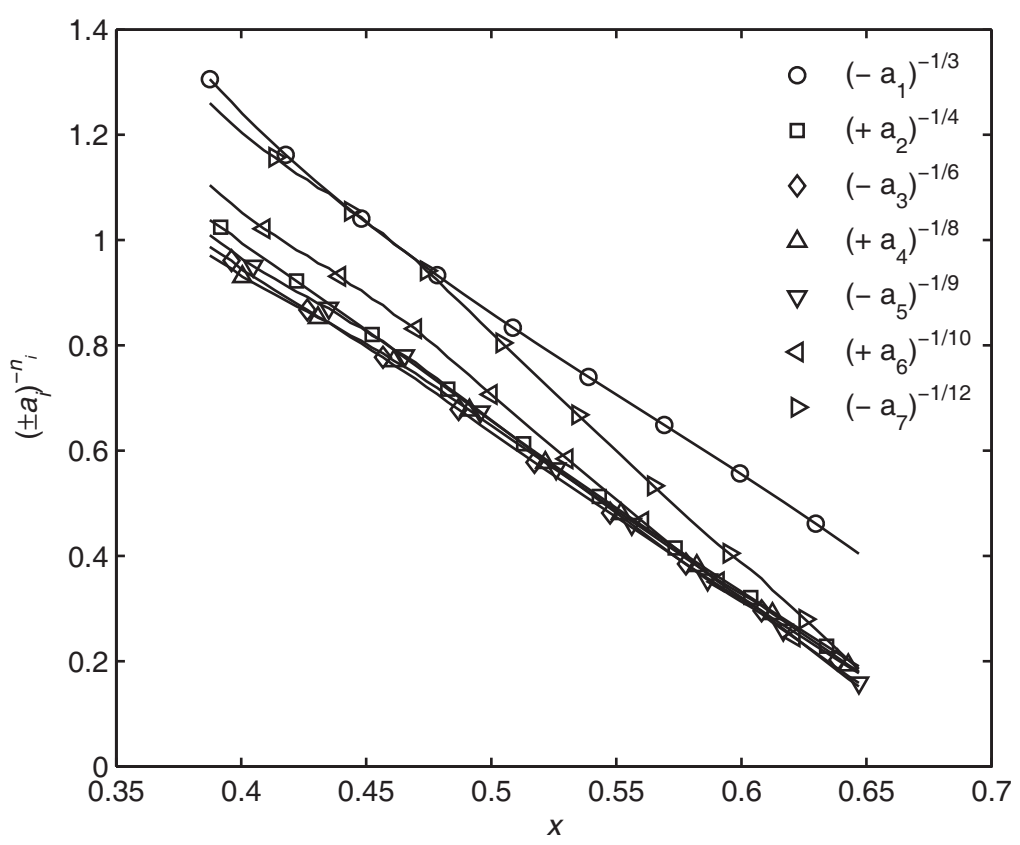

Figure 6: Virial coefficients of Eq. (21) in a scaled plot.

$$
s^{\mathrm{res}}=-R \int_{0}^{\delta} \sum_{i=1}^{7} a_{i}\left(\delta^{\prime}\right)^{i-1}=-R \sum_{i=1}^{7} \frac{a_{i}}{i} \delta^{i} .
$$

Expansion (21) can be related to the conventional virial equation of state

$$
Z=1+\sum_{i \geq 2} B_{i} \rho^{i-1}
$$

The main difference between expansions (21) and (28) is that the conventional virial coefficients $B_{i}$ are functions of temperature, whereas coefficients $a_{i}$ depend on internal energy. Exact relations between coefficients of both series can be found if both series are considered as Taylor expansions about the zero-density point:

$$
\begin{gathered}
a_{1}=\rho_{c} B_{2}, \\
a_{2}=\rho_{c}^{2}\left[B_{3}+\frac{R T^{2}}{c_{v}^{\text {id }}}\left(\frac{d B_{2}}{d T}\right)^{2}\right] .
\end{gathered}
$$

Relations (29) and (30) are valid only when both the constant-temperature expansion and the constant-internal-energy expansion are treated as Taylor expansions. In practice, these are rather polynomial fits to a range of densities. Therefore, the relations are valid only approximately.

\section{Conclusions}

A formulation of thermodynamic properties of superheated, saturated and metastable supersaturated steam was developed. It is primarily intended for application in computational fluid dynamics. Therefore, density and internal energy were chosen as 
independent variables. The present approach combines table look-up (in the $u$-direction) with a global equation (in the $\rho$-direction). Pre-computed table of the ideal-gas entropy at the reference density $s_{\text {ref }}^{\text {id }}$ and seven coefficients $a_{1}, \ldots, a_{7}$ are tabulated in dependence on the internal energy. Quadratic spline interpolation is used, ensuring smoothness of the entropy function, given by Eq. (11), and continuity of the temperature and pressure functions, derived using relations (5) and (6). Quantities depending on second derivatives of the entropy (e.g. speed of sound, heat capacities) vary discontinuously, however in narrow tolerances. Smoothness of temperature and pressure, and continuity of second derivatives, can be provided by using a cubic interpolation method, at the cost of somewhat higher computing time.

The formulation is valid in a range exceeding the operation range of present steam turbines, including high-pressure turbines of supercritical cycles and PWR cycles. The formulation is also valid in the region of metastable supersaturated steam. Unlike the IAPWS-95 and IAPWS IF-97 formulations, both stable and metastable regions are described by a single formulation.

\section{ACKNOWLEDGMENTS}

The author gratefully acknowledges a support by grant No. IAA200760905 of the Grant Agency of the Academy of Sciences of the Czech Republic and grant No. P101/11/1593 of the Grant Agency of the CR. Participation in IAPWS activities was supported by grant No. LA09011 of the Ministry of Education, Youth and Sports of the CR.

\section{RefERENCES}

[1] W. Wagner W., Pruß A.: The IAPWS Formulation 1995 for the Thermodynamic Properties of Ordinary Water Substance for General and Scientific Use. J. Phys. Chem. Ref. Data 31 (2002) 387-535.

[2] International Association for the Properties of Water and Steam, Release on the IAPWS Formulation 1995 for the Thermodynamic Properties of Ordinary Water Substance for General and Scientific Use, available at http://www.iapws.org (September 1996).

[3] Wagner W., Coope, J. R., Dittmann A., Kijima J., Kretzschmar H.-J., Kruse A., Mareš R., Oguchi K., Sato H., Stöcker I., Šifner O., Takaishi Y., Tanishita I., Trübenbach J., Willkommen Th.: The IAPWS Industrial Formulation 1997 for the Thermodynamic Properties of Water and Steam, J. Eng. Gas Turbines \& Power 122 (2000) 150-182.

[4] International Association for the Properties of Water and Steam, Revised Release on the IAPWS Industrial Formulation 1997 for the Thermodynamic Properties of Water and Steam, available at http://www.iapws.org (August 2007).

[5] Miyagawa K., Hill P.G.: Rapid and Accurate Calculation of Water and Steam Properties using the Tabular Taylor Series Expansion Method, ASME J. Eng. Gas Turbines \& Power 123 (2001) 707-712.

[6] International Association for the Properties of Water and Steam, Guideline on the Tabular Taylor Series Expansion (TTSE) Method for Calculation of Thermodynamic Properties of Water and Steam, available at http://www.iapws.org (August 2003). 